half as much to the left hand. The right hand showed no erythema or epilation, but dull pain in the fingers and palm (suggesting intermittent ischaemia due to thrombosis or spasm of small blood vessels) persisted for several months.

Two hours after exposure the patient showed a normal blood count. By four hours the leucocyte count had doubled and the lymphocyte count had fallen from 2,840 to $948 / \mathrm{mm}^{3}$, at which level it remained, with small fluctuations, for four weeks. The platelet count remained at its normal level of about $250,000 / \mathrm{mm}^{3}$ until the 12 th day and then fell sharply, reaching its lowest value, $37,000 / \mathrm{mm}^{3}$ on day 29 . The granulocyte count also fell. The lowest white blood cell count was 2,000/ $\mathrm{mm},{ }^{3}$ recorded on day 36 . The patient was nursed in a sterile laminar airflow unit from day 25 to reduce the risk of infection. No prophylactic antibiotics were given, and his food was not sterilized.

Chromosome studies, though technically difficult, give a useful indication of systemic radiation damage. In the present case sternal marrow aspirated on day 2 showed abnormalities (most often chromatid breaks) in $70 \%$ of the metaphases analysed. By day 19 these abnormalities had almost disappeared. The sperm count was low $(2 \cdot 3$ million $/ \mathrm{ml})$ after 6 months, when slight morphological abnormalities were noted, but returned to 77 million $/ \mathrm{ml}$ after 14 months. The patient complained of fatigue, which lasted for about four months. The inference of muscle damage was supported by the rise, during the first week, in serum creatine phosphokinase and in urinary excretion of purines and pyrimidines, probably resulting from rapid breakdown of cells.

Though fairly heavily irradiated over most of his body, the patient made a full clinical recovery after conservative treatment. The only drug given was oral penicillin to deal with a mouth infection (diagnosed as Vincent's angina) which appeared on day 36. Vodopick and Andrews emphasize that, contrary to popular belief, a single non-fatal total-body irradiation does not generally produce permanent or chronic somatic damage that is clinically obvious. 1 Vodopick, H., and Andrews, G. A., Archives of Enviromental Health, 1974,
28, 53.

\section{Athletes and Electrocardiograms}

The E.C.G. of the well-trained athlete often shows variations from the normal which can be wrongly thought to be due to organic cardiac disease. ${ }^{12}$ Physical examination with electrocardiographic assessment is a prerequisite for participants in an ever-increasing number of sports, and the physician is likely to be presented with an asymptomatic athlete who has an apparently abnormal E.C.G. Because the athlete, professional or amateur, is subjected to extreme mental and physical stress, it is imperative to determine whether or not a sport (which may be a means of livelihood) should be restricted or abandoned altogether.

Lichtman ${ }^{3}$ and his colleagues recently described five such cases and reviewed publications dealing with the E.C.G. findings in over 500 athletes. Generally speaking, clinical abnormalities are absent, but bradycardia, sinus arrhythmia, and a diminished first heart sound may be found. Sinus bradycardia, sinus arrhythmia, and first-degree atrioventricular block are the common resting E.C.G. arrhythmias, ${ }^{3}$ whereas Wenckebach second-degree atrioventricular block, junctional rhythm, and a wandering atrial pacemaker occur rarely ${ }^{4}$ all are the result of increased vagal tone and may be abolished or reduced by exercise and atropine. ${ }^{3}$ Intraventricular conduction defects and incomplete right bundle branch block are extremely common, the latter occurring in about $16 \%$ of athletes. ${ }^{3}$ Rarely the amplitude and duration of the $P$ wave, which may be notched, are greater than normal at rest or after exercise. ${ }^{2}$ The amplitude of the QRS complex in limb and precordial leads may fulfil the voltage criteria for either right or left ventricular hypertrophy, ${ }^{3}$ the latter being present in $76 \%$ of athletes in one series. ${ }^{1}$

Elevation or depression of the ST segment may be found sometimes accompanied by biphasic or inverted $\mathrm{T}$ waves or waves of increased amplitude, but the ST segment usually becomes isoelectric after exercise ${ }^{2}{ }^{3}$; similar abnormalities are found in the general population ${ }^{5}$ and in 5 to $25 \%$ of negroes ${ }^{6}$ (who probably constituted a large percentage of the athletes reviewed). These E.C.G. changes may be mistaken for myocardial ischaemia, and absent $\mathrm{R}$ waves in the precordial leads may suggest anterior myocardial infarction. ${ }^{3}$

The differentiation of this range of E.C.G. variants from the frankly abnormal is not easy. Careful study of the E.C.G. after exercise will generally show abolition or reduction of the abnormality, and this, together with the absence of symptoms and clinical findings, should enable the physician to state with confidence that the E.C.G. changes are not indicative of disease but merely variants of the normal.

\footnotetext{
${ }^{1}$ Smith, W. G., Cullen, K. J., and Thorburn, I. O., British Heart fournal,

1964, 26, 469.
Ganse Van, W., et al., British Heart Fournal, 1970, 32, 160.

${ }^{2}$ Ganse Van, W., et al., British Heart fournal, 1970, 32, 160. 763.

3 Lichtman, J., et al., Archives of Internal Medicine,
4 Grimby, G., and Saltin, S., Lancet, 1964, 2, 962.

Wasserburger, R. H., Alt, W. J., and Lloyd, C. J., American Fournal of Cardiology, 1961, 7, 184.

6 Thomas, J., Harris, E., and Lassiter, G., American fournal of Cardiology, $1960,5,468$.
}

\section{Surgery for Severe Obesity}

Severe obesity is a disabling and life-shortening condition. Reduction in body fat can occur only when calorie intake is less than expenditure. In the great majority of patients this is achieved by increasing physical activity and dietary restriction, which may be aided by the use of drugs to depress appetite. For patients who fail to respond to this regimen and whose degree of obesity threatens life surgical treatment by means of an intestinal bypass should be entertained.

The aim of this operation is to create a malabsorption state that allows normal health but results in the desired reduction of excessive adipose tissue. A jejunocolic short circuit was first introduced. ${ }^{1}$ It resulted in effective weight reduction but was accompanied by considerable metabolic complications. After much experiment and clinical experience Payne and DeWind ${ }^{2}$ recommended that the proximal 14 in $(36 \mathrm{~cm})$ of jejunum should be anastomosed end to side to the terminal 4 in $(10 \mathrm{~cm})$ of ileum, and this procedure is the one most commonly favoured in those units with considerable experience in this type of surgery. Body weight rapidlyfalls within the first few weeks after operation and then reaches a plateau at about one year, by which time an average weight loss in the region of $40 \mathrm{~kg}$ can be expected. ${ }^{3-5}$

R. M. Baddeley ${ }^{6} 7$ reports from Birmingham on the results of this operation on 60 patients followed up for three to 42 months. Four of his cases developed acute fatty enlargement of 
the liver, which was accompanied by lethargy, weakness, and hypotonia. One of these patients died, but the others responded to a high protein, high carbohydrate, and low fat diet. Transient diarrhoea and vomiting were common but usually settled by three months. Persistent diarrhoea occurred in only four of the patients.

Weismann ${ }^{8}$ has reported his experience with 123 patients undergoing jejuno-ileal bypass. This operation was supplemented in 80 cases by a panniculectomy, in which the abdominal apron of superficial fat was excised (a procedure which has been nicknamed "apronectomy"). Two patients died from pulmonary embolism and one patient died six months after surgery with advanced necrosis of the liver. Two patients required reversal of the operation, one because of severe persistent vomiting and malnutrition and the other because of the development of severe ulcerative colitis. A dramatic loss of weight in this series was maintained in patients followed up over two to three years. Careful postoperative metabolic studies showed depression of the serum potassium until diarrhoea came under control. The serum cholesterol was reduced and remained low. The Schilling test gave results well below normal and the glucose tolerance curve was flattened for up to two years or more postoperatively. This last appears to be a satisfactory laboratory assessment of the adequacy of the bypass procedure. Liver biopsy studies carried out in 88 patients at the time of operation showed mild to severe fatty infiltration. Forty patients had subsequent biopsies performed at intervals of 10 days to three years after surgery. The general pattern was of continued fatty infiltration between 6 and 12 months after operation and then a decrease in the subsequent 12 months. No progressive cirrhotic changes were observed. The patients adapted well to the malabsorption state and in the long term only vitamin $\mathrm{B}_{1_{2}}$ must be given indefinitely.

Baber and his colleagues ${ }^{9}$ in their series of 90 patients undergoing bypass had only two deaths, both from liver failure, but two more patients had to have the bypass taken down for progressive liver disease. Their serial biopsy studies also suggest continued progression in the fatty infiltration of the liver for the first year after operation and then improvement.

Schwartz and his colleagues ${ }^{10}$ note that these fairly severe operations can be performed on grossly obese and often poorrisk patients with a reasonably low morbidity and mortality. In their series of 94 cases there was only one death from a perforation of an acute stress ulcer of the colon. As might be expected in this type of patient, wound infection and pulmonary emboli are often reported, and, interestingly enough, stress ulceration of the stomach occurs from time to time. Payne and his colleagues, ${ }^{11}$ with the most extensive experience of this type of surgery, now amounting to 165 bypass operations spread over 16 years, report a $6^{\circ}{ }_{0}$ mortality, a risk which is becoming even lower in recent years. Corso and Joseph ${ }^{12}$ have had no deaths in their 34 operations performed since 1970.

The mortality and morbidity of this operation must be pitted against the poor long-term prognosis of the massively obese patient who fails to respond to the most stringent medical treatment. Though the late results of the operation have still not been fully assessed, particularly the risk of eventual nutritional deficiencies and the development of hepatic cirrhosis, the procedure appears to have a good record for rehabilitation of these unfortunate people back to a useful active life.

\footnotetext{
${ }^{1}$ Payne, J. H., DeWind, L. T., and Commons, R. R., American fournal of
Surgery, 1963, 106, 273.
}

2 Payne, J. H., and DeWind, L. T., American fournal of Surgery, 1969, 118, 14

${ }^{3}$ Schwartz, H., and Jensen, H. E., Acta Chirurgica Scandinavica, 1973, 139, 551.

${ }^{4}$ Hallberg, D., and Backman, L., Acta Chirurgica Scandinavica, 1973, 139, 557.

${ }^{5}$ Scott, H. W., et al., Annals of Surgery, 1973, 177, 723.

6 Baddeley, R. M., British Fournal of Surgery, 1973, 60, 903.

' Baddeley, R. M., Proceedings of the Royal Society' of Medicine, 1973, 66, 1098.

8 Weismann, R. E., American fournal of Surgery, 1973, 125, 437.

${ }^{9}$ Baber, J. C., Hayden, W. F., and Thompson, B. W., American fournal of

Surgery, 1973, 126, 769.
10 Schwartz, M. Z., Varco, R. L., and Buchwald, H., fournal of Surgical Research, 1973, 14, 147.

11 Payne, J. H., et al., Archives of Surgery, 1973, 106, 432. 12 Corso, P. J., and Joseph, W. L., Surgery, Gynecology and Obstetrics,

\section{Conversations on the Social Services}

Some obvious unease about the working of the social services Act was evident at last year's Annual Representative Meeting and clearly reflected widespread concern among doctors. Later in the year a survey by the G.M.S. Committee confirmed that most local medical committees thought that social workers were less co-operative and that their standards of training had fallen since the introduction of the Seebohm proposals, particularly in services for the mentally ill and handicapped. Regional committees for hospital medical services reported that social-work standards had fallen in hospitals; and indeed the new, generic social workers seemed to many doctors to give medicine a low priority.

The Seebohm removal of the social services from medical control caused a massive administrative upheaval against a background of impending large-scale alterations in the Health Service itself. A year after the shout of anger at Folkestone it might reasonably be asked whether things are still as bad, and if so why-a change of attitudes, poor communications, or shortage of people and money. To try and answer some of these questions we start this week a short series of "Medical Practice" articles reporting conversations with various individuals-general practitioners, consultants, and social workers. The first article, giving the views of two rural general practitioners, on their relationships with the sociai services, appears at p. 595.

\section{Attractions at Hull}

In comparison with ten years ago doctors looking for postgraduate teaching sessions have an enormous range to choose from. While it may be simpler for them to confine themselves to occasional lunchtime and evening sessions close to home, there is a lot to be said in favour of getting away for a few days. Talking shop with colleagues from other parts of the country makes a stimulating change from the round of familiar faces at local meetings. This is especially true when an attractive programme is offered, as it is at the B.M.A. Scientific Meeting at Hull next month.

Full details of the sessions are given in the Supplement at p. 107. The main topics are medical intervention-questioning whether treatment is always justifiable; the management of pain; communications in medicine; cancer; and doctors and machines. As usual at these meetings a programme of social and cultural events has been arranged for non-medical members of doctors' families. Application for registration should be sent as soon as possible. 